\title{
Eine Reise nach Phönizien im ll. Jahrhundert v. Chr.
}

\author{
Von Avol.f Erman.
}

Der Reisebericht des Tempelbeamten Wen-Amon, den W.Golenischeff vor einer Reihe von Jahren in Ägypten erwarb, liegt uns jetzt in einer vorlăufigen Vcröffentlichung seines glücklichen Entdeckers vor ${ }^{1}$ ) und zeigt sich als ein kulturgeschichtliches Denkmal, das auch unsere hochgespannten Erwartungen noch übertrifft. Die Übersetzung, die GoLenischefF seiner Ausgabe beigegeben hat, verdient alles Lob; es war eine schwere Aufgabe, diesen Text in vulgärster Sprache mit all den lebhaften Wechselreden als Erster zu interpretieren. Natürlich wird aber ein anderer, der ja die erste Arbeit schon gethan vorfindet, hier noch in den Einzelheiten richtiger übersetzen und damit denn doch auch ein anderes Gesamtbild gewinnen. So glaube ich nichts Überflüssiges zu thun, wenn ich die folgende neue Übertragung hier zum Abdruck bringe ${ }^{2}$ ). Ich hatte sie am 5. April d. J. in der Berliner Akademie vorgelegt; seither ist auch von $W . M_{A x} M_{\text {ÜLLeR }}^{3}$ ) eine Übersetzung des Papyrus veröffentlicht worden, die mich aber nur an einer Stelle (II 61) zu einer Änderung der Ineinen bewogen hat. Im ganzen folgt W. Max MülLER Golenischefrs Interpretation, vor allem auch darin, dafs er wie dieser die Haupterzăhlung (von $I x+1$ an) noch in Dor in der Philistaea spielen lăfst, während sie augenscheinlich an dem Orte spielt, wohin sich cine Reise zum Erwerbe von Bauholz zunăchst richten mufste, am Fufse des Libanon, in Byblos. Ich halte dies für vőllig evident. Auch das als III bezeichnete Bruchstück, das GolensschefF als den Anfang einer dritten oder vierten Seite ansieht, glaube ich in die grofse Lücke der ersten Seite einreihen zu müssen $\left.{ }^{4}\right)$. Bei diesen Änderungen erklärt sich alles ungezwungen; von dem allem, was nach W. Max Mǘler aus dem Papyrus für die Kultur und Geschichte der Philister folgen sollte, bleibt freilich nicht eben viel übrig.

Für Nichtägyptologen bemerke ich noch, dafs die in unserem Text mehrfach genannten -Zakar" (Tkr) uns schon aus den Inschriften Ramses' III. als ein raubendes Barbarenvolk bekannt waren, das zusammen mit den Philistern

1) Rec. de Trav. XXI (auch separat erschienen).

2) Sie beruht nur auf Goleniscrefrs hieroglyphischer Transskription, die aber, wie das von ihm mitgeteilte Faksimile des Anfangs zeigt, bis auf Kleinigkeiten zuverlässig ist.

3) Studien zur vorderasiatischen Geschichte II. Die Urheimat der Philister. Der Papyrıs Goleniscrefr. Die Chronologie der Philistereinwanderung (Mitteil. d. Yorderasiat. Gesellsch. 1900, 1).

4) Nach dem Inhalt mufs ich so urteilen; ob es dem Original gegenüber materiell möglich ist, wird uns nur GoleniscBefF sagen können.

Zeitschr. f. Átyll Spr., XXXVIII. Band. 1900. 
(Prst), den ડ̌̌krs und Wł̌s Nordpalāstina und Āgypten angriff. In unserem Reisebericht (also ein Jahrhundert später) treffen wir dieses Volk angesiedelt und als Besitzer der Stadt Dor an, die südlich von der Spitze des Karmel am Meere lag. Sie hatten sich also in demselben Landstriche festgesetzt wie ihre alten Verbündeten, die Philister, und wenn ihr Name für das alte Testament verschollen ist, so werden wir annehmen dürfen, dafs sie später ganz mit diesen verschmolzen sind.

Die Zeit des Reiselerichts hat Golenischerf schon bestimmt: der Hohepriester Hrihor ist der Priesterkönig dieses Namens, und der Smendes ist der erste König der tanitischen Dynastie. Aber ich möchte noch darauf hinweisen, dafs weder der eine noch der andere hier irgend einen kőniglichen Titel führt; Hrihor ist einfach der Hohepriester des Amon, Smendes und die Frau TentAmon ${ }^{1}$ ) sind - die Befehlshaber (?), die Amon dem Norden seines Landes gegeben hat:, daneben existieren noch andere grofse Fürsten «. Das macht es für mein Gefühl unwahrscheinlich, das Datum des -Jahres 5 ^, mit dem der Papyrus beginnt, auf Hrihor zu beziehen; es wird vielmehr das Jahr des letzten Ramessiden sein, von dem wir ja ohnehin angenommen haben, dafs er nur noch als Schattenkönig unter Hrihor regiert habe. Nur einmal ist in unserem ganzen Texte von dem regierenden -Pharao. die Rede und auch dies geschieht nur in der spöttischen Bemerkung eines ägyptischen Dieners am phönizischen Hofe; es kőnnte wohl eine besondere Bosheit ir seiner Erwăhnung liegen ${ }^{2}$ ).

Dafs der Bericht wirklich so erstattet ist, glaube ich auch ${ }^{3}$ ); ich möchte ihn sogar für das Original oder die aktenmärsige Kopie halten $\left.{ }^{4}\right)$. Den Grund, weshalb Wen-Amon sich so ausführlich über seine Reise āufsert, hat W. MAx MüLLER gewifs richtig dahin angegeben, dafs er sich bei dem ungenügenden Erfolge des Unternehmens rechtfertigen wollte: er hatte ja nicht viel erreicht, aber wie sollte er auch etwas erreichen ohne genügendes Geld, ohne Empfehlungsbriefe, ohne Schiffe? Denn das Götterbild, das man ihm zum Reisegenossen mitgegeben hatte und das Heil und Segen mit sich brachte, hatte dem Barbaren wenig Eindruck gemacht - dem wäre vieles Geld lieber gewesen -,

1) Diese Mitregentin des Smendes gilt dem Boten des Hrihor offenbar als eine sehr wichtige Person; man könnte denken, dafs sie eine an Smendes verheiratete Angehörige des Hohenpriesterhauses gewesen sei.

2) Dafs die Bemerkung sehr boshaft sein ınufs, sieht man daraus, dafs sie auch dem Phönizierfürsten zu stark ist; er weist den Diener zurecht.

3) Ich vermisse in dem Berichte nur eins: wie unten gezeigt, hat Wen-Amon dem Fürsten von Byblos schliefslich ein Zahlungsversprechen gemacht; das wird aber nicht so vag gehalten gewesen sein wie in unserem Text, sondern auf eine bestimmte Summe gelautet haben. Aber unser Bericht war wohl nur der Generalbericht über das Unternehmen; daneben wird in einem Spezialbericht angezeigt worden sein, wieviel Holz erworben war und was dafür zu zahlen blieb.

4) Die Schreibfehler und Auslassungen, die nach W. Max Mǘs.fr diese Annahme unmōglich machen sollen, sind nach meinem Gefühl um nichts zahlreicher als in jedem ägyptischen Text, denn die Fortlassung der Präpositionen darf man nicht dazu rechnen, sie zeigt nur, dafs WenAmon kein gelehrter Schreiber ist. 
und auch den Hinweis auf Ägyptens altes Verhältnis zu seinem Lande hatte dieser nur mit einem Hinweis auf die Zahlungsfahigkeit der früheren Pharaonen beantwortet. Dazu noch der Diebstahl in Dor, der schlechte Wille des dortigen Fürsten und was sonst noch an Unglück geschah - es war begreiflich, dafs eine solche Reise keinen glatten Erfolg ergeben hatte.

Es ist ja natürlich unmöglich, nach dreitausend Jahren noch über Recht und Unrecht in einer solchen Sache zu urteilen, aber ich mőchte doch sagen, dafs mir Wen-Amons Darstellung nicht unglaubwürdig aussieht; er hătte sie ja auch nicht so abfassen kőnnen, wenn nicht der Hauptpunkt, seine mangelhafte Ausstattung, ein unleugbares Faktum gewesen wäre. Ist dem aber so, dann giebt uns sein Bericht auch ein Bild für den traurigen Verfall Ägyptens in dieser Zeit der staatlichen Zerrüttung.

Der thebanische Priesterstaat hat zwar noch die Bedürfnisse der früheren Jahrhunderte, aber keine Mittel mehr, um sie zu befriedigen; er ist verarmt und ist zudem für den Verkehr mit dem Auslande auf den guten Willen des Deltafursten angewiesen. Und dieser gute Wille reicht eben auch nicht allzu weit; Smendes entsendet den Gesandten des Amon nicht auf einem seiner Hauptschiffe, das man auch in Phönizien als das seine erkennen würde, sondern setzt ihn auf das Schiff eines fremden Kapitäns, das dem Smendes untergeben sein mag, das aber die Phönizier für ein privates phönizisches Schiff ansehen müssen und das nicht einmal seine Rückkehr abwartet. Und wie traurig steht es nun erst mit Ägyptens Einflứs im heiligen Lande; seine Vorherrschaft ist völlig dahin, und nicht ohne Mitgefuhl lesen wir, wie schnöde sich der arme ägyptische Gesandte von den dortigen Dynasten liehandeln lassen murs. Ägypten hatte bei diesen wohl noch seinen Nimbus als das Land der höheren Kultur und Bildung (wie das der Fürst von Byblos selbst dem Wen-Amon versichert), aber zu fürchten brauchte man es nicht mehr.

Ich habe geschwankt, ol, ich dieser Arbeit eine grammatische Skizze dieses vulgärsten aller neuăgyptischen Texte ${ }^{1}$ ) beigeben sollte; ich habe es schliefslich unterlassen, weil ich ohnehin diese Untersuchungen in gröfserem Zusammenhange aufnehmen möchte. Aber auf einige Erscheinungen mufs ich doch hier kurz hinweisen, da sie meine Übersetzung vielfach bestimmt haben.

Das Pseudopartizip ist bis aufs äufserste reduziert; es besitzt nur noch die Formen sodm und sdomt, deren letztere auch für die 1. sg. gilt.

Wie Sethe nachgewiesen hat $^{2}$ ), hat das Neuägyptische die emphatische Form der Konjugation durch die Umschreibung mit $40_{\infty}^{\infty}$, der emphatischen Form von espe ersetzt. Der Verfasser unseres Textes benutzt diese Form mit besonderer Vorliebe, manchmal schon fast wie ein Ачссштм.

1) Nur die -Maximes d'Anii - kommen ihm gleich, deren nahe Verwandtschaft auch Gotensschf.FF mit Recht hervorlieht.

2) Strra, Das Ägyptische Verbun II, $\$ 206.350$. 
Ich habe früher schon') darauf hingewiesen, dafs stark vulgăre Handschriften hăufig die Prăpositionen $\overline{\mathbf{n}}$, $\in$ und $q^{1}$ sowie das genetivische $\overline{\mathbf{n}}$ unhezeichnet lassen. Der Schreiber unseres Papyrus geht darin so weit, wie ich es nur noch bei dem der Maximes d'Anii kenne:

Er unterdrückt das ${ }_{1}$ in der mit dem Infinitiv zusammengesetzten Verbalform ausnahmslos. Er unterdrückt es auch sonst vor dem Infinitiv (I $x+10$ : II 3. 25. 39. 47. 64. 67).

Er unterdrückt sogar $\bigcirc$ im Futurum (II 33).

Er unterdrückt $\bigcirc$ mehrfach vor Substantiven: •ich ging [zum] Strande * (II 62; auch II 44. 58).

Er unterdrūckt (1 22; auch $I x+24)$; - geh heraus [aus] meinem Hafen. (I $x+3 ; x+9)$; - einer [von] seinen Jünglingen. (I $x+3)$; - ich stattete ihn aus [mit] meinen Schiffen. (II 57). Andere Beispiele: I $x+13$; II 14. 52. 59).

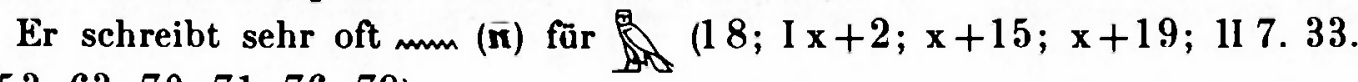
52. 53. 63. 70.71. 76. 79).

Er unterdrückt das dativische mun (I $x+3$; II 17. 30. 71).

Er unterilrückt das genetivische amm (I 21 sogar in gengoor; II 8. 32).

In der hier folgenden Übersetzung sind die Zeilen des Originals von 5 zu 5 angegeben.

$11 \mathrm{~lm}$ Jahre 5, im dritten Sommermonat, am 16. Tage, an diesem Tage reiste Wen-Amon, śmsis- $h 3 y t^{2}$ ) der Verwaltung des Amon [von Karnak?] ${ }^{3}$ ) ab, um das Holzwerk zu holen für das grofse, herrliche Schiff des Amon-Re, des Götterkönıgs, das sich auf ... befindet, Wis-h(t-Amon $\left.{ }^{4}\right)$.

An dem Tage, wo ich in Tanis ankam, am Wohnorte des Smendes und der Tent-Amon, übergab ich ihnen die Schreiben des Amon-Re, des Götterkönigs. Sie ${ }^{5}$ liefsen sie sich vorlesen und sagten: -Ja, ich thue nach dem, was Amon-Re, der Götterkönig, unser Herr, sagt..

Ich blieb bis zum vierten Sommermonat in Tanis. Smendes und TentA mon sandten mich zusammen mit dem Schiffskapităn $M n g b t^{5}$ ) ab, und ich fuhr am Ersten des vierten Sommermonats zum grofsen syrischen Meere herab.

Ich kam nach Dor"), einer Zakar-Stadt, und ihr Fürst $B d r$ liefs mir viele Brote, ein $m s h$ Wein ${ }^{10}$ und eine Rinderkeule bringen ${ }^{\top}$. Ein Mann von meinem Schiff entfloh, indem er folgendes stahl:

1) ÄZ. 1896, S. 154 .

2) Was dieses uralte Amt in dieser späten Zeit zu bedeuten hatte. ist mir nicht beknnnt

3) Die Ergänzungen Golenischfrfs in Z. 2 und 3 sind zu grofs für die Lïcken.

4) So nennt unser Text das Götterschiff des Aınon auch 1125.

b) $M-n-g 3-b w-t i$.

o) Die Namen sind $D-r, T 3-k_{j-r}$ und $B i-d i-r$ geschrieben.

$\left.{ }^{7}\right)$ Als Gastgeschenke; als Quantităt der Brote ist wörtlich 10000 angegeben. 


\section{Gold [Gefafse] . . . (an Gewicht) $5 \mathrm{Dbn}$ \\ Silber, 4 Gefärse, . . . . . . $20 \mathrm{Dbn}$ \\ Silber (in einem) Sack ${ }^{1}$ ) .... $11 \mathrm{Dbn}$ \\ [Zusammen, was] er [stahl]: $5 \mathrm{Dbn}$ Gold und \\ $31 \mathrm{Dbn}$ Silber ${ }^{2}$ ).}

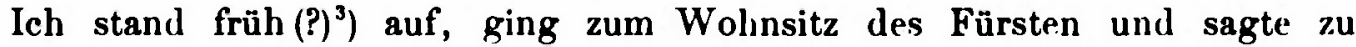
ihm: Ich bin in deinem Hafen bestohlen worden. Du bist doch der Fürst dieses Landes und du bist doch sein Richter ${ }^{4}$ ), so suche du mein Geld. Wahrlich, das Geld gehört dem Amon-Re, I 15 dem Götterkőnig, dem Herrn der Länder, es gehört dem Smendes, es gehört meinem Herrn, dem Hrihor und den anderen grofsen Fürsten Ägyptens ${ }^{5}$ ); es gehört ferner dem $W_{r t^{\circ}}$ ), es gehört dem $M k m r$, es gehört ‘’em Zekarbac ‘l, dem Fürsten von Byblos *.

Er sagte zu mir: - Deinem Zorn und deiner Güte(? $\left.{ }^{7}\right)$. Aber sieh, ich weils nichts von dieser Geschichte, die du mir sagst. Wenn der Dieb, der in dein Schiff gekommen ist und dein Geld gestohlen hat, aus meinem Lande wäre, so hätte ich es dir aus meinem Schatz erstattet, bis man 20 den Namen deines Diebes ermittelt hătte. Nun gehört aber der Dieb, der dich bestohlen hat, doch zu deinem Schiff. So bleibe einige Tage hier bei mir, dals ich ihn suche .

Ich verbrachte neun Tage, indem ich in seinem Hafen lag. Ich ging zu ihm und sagte zu ihm: - Sieh, du hast mein Geld nicht gefunden, [so lafs mich abreisen?] mit [dem?] Kapităn und mit denen, die .... gehen ...*

Hier beginnt die grolse Lücke, an deren Schlufs ich das von Goleniscarff als Ill bezeichnete Fragment setze; auch so fehlen immer noch ca. 23 Zeilen ganz, und auch die Zeilen des Fragmentes sind grofsenteils zerstört. Ich gebe von diesen die verständlichen Worte und ergänze den $\mathrm{Zu}$ sammenhang, so gut es geht.

III 1... Er sagte zu mir . Schweige .....

Es folgen drei Zeilen, in denen noch vom Suchen der Diebe die Rede ist, offenbar mit Bezug auf den Diebstahl in Dor.

1) Das heifst kleinere Silbersachen, Bruchsilber u. s. w.

2) $455 \mathrm{~g}$ Gold und $2,821 \mathrm{~kg}$ Silber.

3) Ob steht? Die Lesung $\triangle \mathbb{R}_{\text {L }}$ sist wohl irrig.

4) Wörtlich -Untersucher..

5) Inwiefern das Geld des Amon auch dem Hrihor, Smendes und den anderen Fürsten gehört, ist nicht klar; vielleicht meint er, dafs diese Herren es dem Amon gespendet haben zur Herstellung seiner Barke. Die dann noch genannten Nichtägypter sind wohl die Fürsten, für die das Geld bestimmt war, also die, zu denen Wen-Amon gesendet ist.

9) W3-r-ti, $M-k 3-m-r v$ und $T_{3}-k 3-r w-b c-r$. Dals der letztere Name brot ist, hat auch W. Max Müller gesehen.

7) Scil. füge ich mich. So auch Golenıscheff; es mufs eine ägyptische Höflichkeitsphrase sein. 
II 5... der Hafen ........ Tyrus. Ich ging aus 'Tyrus heraus bei Morgengrauen ...

Er hat also seine Reise fortgesetzt, vermutlich auf dem Schiffe, das er vorher benutzt hat; er fahrt weiter, uin z.u

Zekarbałal, dem Fürsten von Byblos, zu kommen.

Irgendwie trifft er mit Zakarleuten zusammen, die wohl einen Sack Geld bei sich halsen, und er mag denken, sich an diesem schadlos zu halten für das Geld, das ihın in Dor gestohlen ist ').

[Ich offnete ihn?] und ich fand 30 Deben Silber darin. Ich ergriff es [und sagte zu ihnen: Ich nehme] euer Geld, es bleibt bei mir, bis ihr [mein Geld] finden werdet ${ }^{2}$ ). [Denn ihr sagt, ihr wilst nicht, wer] ${ }^{10}$ es gestohlen hat; kein Dieb(?) ......, so werde ich es fortnehmen .......

Nach einer kleinen Lücke folgt dann:

Sie gingen fort; ich ........ gelangte ....... dem Hafen von Byblos.

Er geht in Byblos ans Land und verbirgt dort irgendwo seine Halse"):

[Ich verbarg in einem ....] den Amon des Weges ${ }^{\downarrow}$ ) und ich legte seine Sachen in es hinein.

Der Fürst von Byblos liefs mir sagen: 'Mach' dafs du aus meinem Hafen kommst $\left.{ }^{5}\right)$.. Ich liefs ihm sagen:.$\ldots \ldots$....

Hieran schlielst nach einer nicht grolsen Lücke der Schluls von Blatt I; die ersten erhaltenen Worte gehören noch z.ı der Verhandlung mit dem Fürsten von Byblos, die diesen, wie man aus dem Folgenden sieht, zur Genüge über die Lage des Boten des Amon und das, was er bei sich hat, unterrichtet. Er weigert sich daher, den Wen-Amon und seinen Gott zu emplangen. Der arme Gesandte würde auch gern nach Ägypten zurückkehren, aber das Schiff, das ihn nach Byblos gebracht hatte, ist offenbar wieder fortgefahren.

Ix+Ix. . . wenn man fährt, so möge man mich(?) nach Ägypten führen(?) *.

Ich verbrachte 19) Tage in seinem [Hafen], und täglich liefs er mir sagen: - Mach', dafs du aus meinem Hafen kommst..

Als er nun seinen Göttern (einmal) opferte, so ergriff der Gott einen von seinen grolsen Jünglingen; er machte ihn rasend(?) $\left.{ }^{b}\right)$, und er sagte: 'Bring'

1) Diese fragwürdige Selbsthilfe wird es auch sein, weshalb die Zakarleute nachher ihn verhaften wollen (II 62 ff.).

2) Nach щya - bis= steht nicht nur der Konjunktiv (I 19; 1166 mit Kontraktion ugator-, wate-), sonderu auch (hier sowohl wie II 36) die durch 4 umschriebene emphatische Form des Verbums, die ja auch sonst nach Präpositionen steht.

3) Dies ergiebt sich aus deln Folgenden $(1 x+7)$.

4) Dafs dies ein Götterbild ist, das er bei sich hat, hat Golenischeff schon erkannt (vergl. seine Anmerkung z.u II 55).

5) Die Stelle muls im Original teilweise schlecht erhalten sein, doch schlägt auch Gor.ENischfFF diese Lesung vor. Übrigens würde man die erhaltenen Worte ohnehin nicht wohl anders ergänzen.

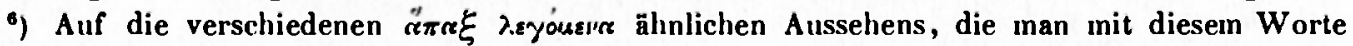
uswot vergleichen könnte, will ich hier nicht eingehen; um was es sich ungefähr handelt, sieht man schon aus dem Determinativ $\mathcal{H}^{\prime}$. Jedenfalls gerät der Knabe in Verzückung, er wird ein x'בי ein -Prophet. und verlangt in seiner Ekstase, dafs der Gott und sein Begleiter nicht lănger schlecht behandelt werden. Und der Fürst wird durch diese Offenbarung des göttlichen Willens 
[den Gott]') hinauf! Bring den Boten, der ihn bei sich hat, I x+5 zu Amon! Sende ihn ab, lasse ihn gehen!*

Als der Rasende(?) so in dieser Nacht raste(?), wăhrend ich (gerade) ein Schiff gefunden hatte, das nach Ägypten gerichtet war, und hatte all das Meinige in es geladen und sah nach der Dunkelheit aus und sagte: "Wenn sie eintritt, so lade ich (auch) den Gott ein, so da(s ihn kein anderes Auge sieht $\alpha^{2}$ ), da kam der Hafenvorsteher zu mir und sagte: Bleibe bis morgen zur Verfugung ${ }^{3}$ ) des Fürsten«. Ich sagte ihm: - Bist du es nicht, der täglich zu mir gekommen ist und mir gesagt hat: 'Mach', dafs du aus meinem Hafen kommst?' * Sagst du nicht heute: -Bleibe «, $\mathbf{x + 1 0}$ damit das Schiff, das ich gefunden habe, abreist? und (dann) wirst du kommen und wirst wieder sagen: - Beeile dich!«

Da ging er und sagte es dem Fürsten, und der Fürst sandte \%u dem Kapitän des Schiffes und liefs ihm sagen: Bleibe bis morgen zur Verfügung des Fürsten..

Als es Morgen geworden war, so schickte er und fuhrte mich hinauf, als das Gottesopfer ${ }^{4}$ ) in der Festung war, in welcher er sich aufhält, am Meeresufer. Ich traf ihn, wie er in seinem Obergemach ${ }^{5}$ ) sals, indem sein Rücken an einem Fenster lehnte, und die Wellen des grofsen syrischen Meeres schlugen gegen .... ${ }^{\mathrm{x}+15}$ hinter ihm $^{\circ}$ ).

Ich sagte zu ihm: Milde(?) des Amon! Er sagte zu mir: - Wie lange ist es bis heute her, seit du vom Wohnsitz des Amon fortgegangen bist? * Ich antwortete ihm: - Fünf Monate und einen Tag bis heut.. Er sagte zu mir: -Sieh, bist du wahrhaft? Wo ist denn das Schreiben des Amon, das du bei dir hast? Wo ist denn der Brief des Hohenpriesters des Amon, den du bei dir hast?* Ich antwortete ihm: -Ich gab sie an Smendes und Tent-Amon *.

Er wurde sehr ärgerlich und sagte zu mir: *Nun sieh, Schreiben und Briefe hast du (also) nicht ${ }^{7}$ ). Wo ist denn (wenigstens) das Schiff aus Akazien(?)-Holz,

umgestimmt. Was der Ägypter uns nüchtern erzählt, ist für uns als ältestes Beispiel der alttestamentlichen Prophetie, von grolsem Interesse. - Auch W. MAx MüLLER falst das Wort als -Verzückung.

1) So ergänzt auch GoLenischeff in der Anmerkung zu II 55. Der Sinn wird sein: lass das Götterbild zu dir herbringen und weise seinen segenspendenden Besuch nicht ab; dann thue weiter auch dem Begleiter des Gottes seinen Willen. - Dals das Götterbild dem Fürsten von Byblos Segen bringen sollte, geht aus II 29. 30 hervor.

$\left.{ }^{2}\right)$ So schwerfallig ist diese Periode auch im Original; Wen-Amon ist kein grolser Stilist.

3) Oder: in erreichbarer Nähe (wörtlich: angesichts).

4) Diese Notiz giebt gleichzeitig an, wohin Wen-Amon geholt wurde und wann dies geschah (zur Zeit des Frühopfers).

3) $c r-t i$ ist offenbar $\rightarrow$ so.

d) Einen äufseren Grund zu dieser Bemerkung sehe ich nicht; sie scheint lediglich durch die Erinnerung an das hübsche Bild veranlafst zu sein.

${ }^{7)}$ In diesem und folgenden Sätzen hebt der Fürst die mangelnde Legitimation des WenAmon hervor und spricht den freundlichen Verdacht aus, er sei vielleicht ein Staatsverbrecher, 
das dir Smendes gegeben hat? Und wo ist denn $1 \mathbf{1 x + 2 0}$ seine syrische Mannschaft? Er wird dich (doch) nicht diesem Kapitän ..... übergeben haben, damit sie dich tőten und dich ins Meer werfen? Von wem hătten sie den Gott gewollt ....? Von wem haben sie dich gewollt? So sagte er zu mir.

Ich sagte zu ihm: -Doch ist es ein $\left.{ }^{\prime}\right)$ ägyptisches Schiff, und es ist auch eine ägyptische Mannschaft, die für Smendes rudert. Er hat keine syrische Mannschaft. Er antwortete mir: "Es liegen doch 20 Schiffe hier in meinem Hafen, die mit Smendes in Verbindung (? $\left.)^{2}\right)$ sind. Und in diesem Sidon, $\mathbf{\|} \mathbf{1}_{\mathbf{7 . 1 1}}$ welchem du auch hin wolltest(?), sind doch auch 10000 Schiffe, die mit Wrktr ${ }^{3}$ ) in Verbindung sind und zu seinem Hause hinfahren:. Ich schwieg in diesem grofsen Augenblick. Er antwortete und sagte zu mir: - Wegen welches Auftrags bist du hierhergekommen?* Ich sagte zu ihm: "Ich bin nach dem Holzwerk des grofsen herrlichen Schiffes des Amon-Re, des Götterkönigs, gekommen. Dein Vater hat (es) gethan $\mathbf{5}^{5}$ und dein Grofsvater hat (es) gethan, so wirst du es auch thun.. So sagte ich zu ihm.

Er sagte zu mir: "Sie haben es wirklich gethan. Du wirst mir (etwas) dafïr geben, dafs ich es thue und so werde ich es (auch) thun. Gewifs, die Meinen haben diesen Auftrag ausgeführt, aber der Pharao schickte auch sechs Schiffe her, die mit ägyptischen Waren beladen waren; man lud sie in ihre Speicher aus. Du sollst auch mir etwas bringen..

Er liefs Tagebücher seiner Väter holen und liefs sie mir vorlesen, und man fand, dafs es $1000 \mathrm{Dbn}^{4}$ ) von allerlei Silber waren, was in seinem Buche stand.

${ }^{10}$ Er sagte zu mir: - Wăre der Herrscher von Ägypten der Herr meines Eigentums und wäre ferner ich auch sein Diener, so hätte er nicht Silber und Gold bringen lassen ${ }^{5}$ ), als er sagte: -Thue den Befehl des Amon*. Es war kein Herrschaftsauftrag $\left.(?)^{6}\right)$, was sie meinem Vater thaten. Ich aber, ich, ich bin weder dein Diener noch der Diener dessen, der dich geschickt hat. Schreie

den man mit Beihülfe des Kapitäns Mnght habe beseitigen wollen. Die Schlufssätze verstehe ich nicht; der Gott ist natürlich das Götterbild, zu wiłh mdr wsuchen von jemand. vergl. Il $58 d b h$ $m d r$ verbitten von jemand , sowie Il 28 ir suov $m d r$. Handel treiben mit jemand.

1) Die doppelten Verneinungen ${ }^{2}$ ( 过 $(1 \mathrm{x}+23 ; 111 ; 1177)$ dienen alle zur starken Bejahung.

2) Das hier gebrauchte Wort bbr ist vielleicht ughpp erhalten.

3) Gewifs ein b-nos; es muls ein in Tanis ansässiger Grofskaufmann sein. - Was der Fürst mit seiner Bemerkung will, sehe ich nicht.

4) $91 \mathrm{~kg}$.

b) Nämlich meinen Vătern.

6) Fy-mrk; in dem fremden $m r k$ steckt gewirs jor. Der Sinn ist: wir sind auch früher nicht eure tributpflichtigen Vasallen gewesen, das siehst du ja daraus, dals ihr uns das Holy bezahlen muistet. 
ich zum Libanon'), so öffnet sich der Himmel, und die Bäume liegen hier am Strande des Mecres"). Gieb $\mathbf{n} 15 \mathrm{mir}$ die Segel, die du (doch gewifs) mitgebracht hast ${ }^{3}$ ), um deine Schiffe, die mit deinem Holz beladen sind, nach |Ägypten|') zu führen. Gieh mir die Stricke ......... bäume, welche ich falle,

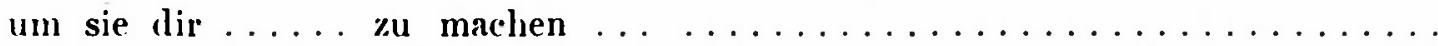
$\ldots \ldots \ldots \ldots \ldots$ ich mache sie dir für(?) die Segel deiner Schiffe, und die Spitzen ${ }^{5}$ ) sind (\%u) schwer und sie zorbrechen, und du stirbst inmitten des Mecres. Sieh, Amon donnert (ja) am Himmel, indem er den Sutech in seine Zeit setzt $\left.{ }^{f}\right)$.

Denn Amon ${ }^{20}$ sorgt(?) für alle Iäncler, er stattete sie aus, indem cr \%uerst das Land Ägypten, aus dem du herkommst, ausstattete. Denn das Treffliche kam aus ihm bis zu meinem Wohnort, und die Ielhre kam aus ihm bis \%u meinem Wohnort $^{\overline{7}}$ ) — was soll (da) diese jämmerliche(?) Reiserei ${ }^{8}$ ), die man dich machen lä̊st?.

Ich antwortete ihm: -Schändlich! Gieht es denn wohl(?) jämmerliches(?) Reisen bei dem, wozu ich gehöre?)? Es gieht ja kein Schiff auf deın Strom, das nicht Amon gehörte. Er ist das Meer und er ist der Libanon, von dem du sagst, du seiest es. $\mathbf{E r}^{10}$ ) ist 25 cin Isandstrich für die Barke Wsr-het-Amon, den Herrn aller Schiffe. Wahrlich, so hat er, Amın-Re, der Götterkönig, gesprochen, indem er zu Hri-hor, meinem Herrn, sagte: "Sende mich."), und so hat er mich mit diesem grolsen Gotte ${ }^{1-}$ ) ausgeschickt. Nun aber sieh, du hast diesen grolsen (iott diese 2!) Tage verbringen lassen, indem er in deinem Hafen gelandet war, obschon du wohl wu(stest(?) $\left.{ }^{1: 3}\right)$, dafs er hier war. Er ist

1) Rbrn mit dem Artikel gebraucht und als Wald determiniert.

2) Der Sinn ist: Wollte ich euch Holz geben, so wäre mir das freilich ein leichtes, denn auf ineinen Befehl rollt das Holz gleich bis an den Strand. Auf diese Renommage erteilt ihm Wen-Aınon dann 1124 die gebührende Antwort.

3) Sinn der folgenden Sätze: Und wenn ich nun euch wirklich Holz verabfulgen wollte, womit wolltest du es denn transportieren:' Du hättest doch zum mindesten für genügende Segel und Stricke sorgen mũssen, denn grofse Stämme lassen sich nicht ohne besondere Vorrichtungen verschiffen; sonst gehst du ja beim ersten Sturme unter.

4) Vom Schreiber ausgelassen.

5) Vergl. Il 38, wonach es die grolsen Blöcke für Vorder- und Hintersteven sein werden.

a) Wohl nur ironische Ausführung der Warnung vor dem Sturm: dein Gott und der meine, der ihın untergeben ist, erregen ja doch zuweilen Stürme. Diese Nennung des Aınon bringt ihn dann auf eine ganz andere Gedankenreihe.

7) Er meint: wir verehren İgypten als das Land, aus dem wir Kunst und Technik und höhere Bildung erhalten haben, da ist doch ein solches Auftreten unter eurer Würde. So fasst es auch Golenischerf.

8) Diese srog-gänge; sicgs mufs nach dem Determinativ etwas Elendes sein.

?) Das heifst wohl nach dem Folgenden: bei dem Amon-Tempel.

10) Der Libanon.

11) Amon hat also durch einen Orakelspruch verkündet, dafs man eins seiner Bilder zaum Abholen des Holzes entsenden solle.

12) Dem Götterbild.

13) Wörtlich: •indem du nicht wufstest, er sei nicht hier*

Zeitzechr. $\mathcal{C}$ Ägy pt. Spr., XXXVIL. Band. 1900. 
noch derselbe, der er gewesen ist ${ }^{1}$ ), und (doch) stehst du da und machst Geschăfte wegen des Libanon mit Amon, seinem Herrn. Wenn du nun aber sagst: -Die früheren Könige haben Silber und Gold geschickta - wenn sie Leben und Gesundheit $\left.{ }^{2}\right)$ geschenkt hătten, so hătten sie nicht die Sachen geschickt! II ${ }^{30}$ Sie haben (eben) statt des Lebens und der Gesundheit deinen Vătern die Sachen geschickt. Nun aber Amon-Re, der Götterkőnig, der ist der Herr des Lebens und der Gesundheit; er war der Herr deiner Văter, und ${ }^{3}$ ) sie brachten ihre Lebenszeit zu, indem sie dem Amon opferten, und auch du, du bist (auch) ein Diener des Amon. Wenn du zu Amon - Ja, ich thue esa sagst und seinen Befehl ausrichtest(?), so wirst du leben und heil sein und gesund sein und wirst deinem ganzen Lande und deinen Leuten angenehm sein.

Wünsche dir nichts, was Amon-Re, dem Götterkőnig, gehört, wahrlich: ein Löwe liebt seine Habe $\left.{ }^{4}\right)$.

Lasse mir meinen ${ }^{5}$ ) Schreiber holen, damit ich ${ }^{36} \mathrm{ihn}$ zu Smendes und TentAmon sende, den Befehlshabern(?), die Amon dem Norden seines Landes gegeben hat, und damit sie dir alles schicken, weswegen ich ihn zu ilınen senden werde, dafs es gebracht werde ${ }^{\circ}$ ), bis dafs ich auch ${ }^{7}$ ) nach Süden kommen, und dir dein elendes Zeug $^{8}$ ), alles, alles, schicken werde. So sagte ich zu ihm.

Er legte meinen Brief in die Hand seines Boten; er lud die pipit, die Spitze des Vorderteils und die Spitze des Hinterteils samt vier anderen behauenen ${ }^{9}$ ) Hölzern (im ganzen sieben) auf und liefs sie nach Ägypten bringen ${ }^{10}$ ). Sein Bote reiste nach Ägypten ab und kam im ersten Wintermonat zu mir nach Syrien zurück. Smendes und Tent-Amon sandten:

40 an Gold: $4 T b-$ und $1 K 3 k$-mnt-Gefă $s$,

an Silber: 5 Tl-Gefässe,

1) Also ist Amon für die Leute von Byblos einst ein angesehener Gott gewesen, wie das auch gleich nachher ausdrücklich gesagt wird. Überhaupt deutet hier alles auf alte Bezielungen zu Ägypten, und in der That sind diese gerade bei Byblos auch sonst als uralt nachzuweisen.

2) Den himmlischen Segen -Leben und Gesundheit. bringt das Götterbild mit sich; die -Sachen: sind das fehlende Geld.

2) Es fehlt wohl ivo.

4) Vielleicht ein Sprichwort. Der Satz soll wohl sagen: Amon würde es rächen, wenn du sein Eigentum, die Cedern des Libanon, ihm vorenthieltest.

3) Lies -deinen.?, denn Wen-Amon hat doch wohl keinen Schreiber bei sich, und weiterhin wird der Bote als der des Fürsten bezeichnet.

6) Wörtlich: -indem ich sage: es möge gebracht werden.

7) $\rightarrow$ bedeutet in unserem Texte wiederholt sicher sauch. (so 11 5.10.13. 32); die Stellung ist die gleiche wie die von gr auch., am Ende des ganzen Satzes.

s) Wörtlich: -dein Jämmerliches -, d.h. das Geld, dem du den Vorzug giebst. Wen-Amon bequemt sich also nach allen grossen Reden zu einem Kompromifs: von Tanis besorge ich dir eine Anzahlung, den Rest schicke ich später aus Theben.

2) Lies 98 $3 k h$.

$\left.{ }^{10}\right)$ Der Fürst schickt diese Balken (die er also vorrătig liegen hat), um auch seinerseits guten Willen zu zeigen. 
an Königsleinen : 10 Stück zu (?) $10 \mathrm{hm}$-h̆rd(?),

feines Papier: 500,

Ochsenhäute: 500 ,

Stricke: 500 ,

Linsen: 20 Sack,

Fische: 30 msts.

Sie sandte') mir:

Leinen: 5 [Stück zu(?)] $5 h m$ - $h r d(?)$,

Linsen: 1 Sack,

Fische: 5 msts.

Der Fürst freute sich und stellte (?) 300 Mann und 300 Ochsen an und setzte Aufseher an ihre Spitze, damit sie die Băume fallten. Sie fallten sie und verbrachten die..$^{2}$ ) Winterjahreszeit damit. Im dritten Sommermonat aber zogen sie sie an den Strand Jes Meeres. Der Fürst ging aus und trat zu ihnen. Er liefs mir sagen: 4 40,Kommes. Als ich nun vor ihn trat, so fiel der Schatten seines Wedels $^{3}$ ) auf mich, und Pen-Amon, ein Truchsels, der trat zwischen mich und sagte: -Der Schatten des Pharao, deines Herrn, fallt auf dich . $\left.{ }^{4}\right)$. Er wurde auf ihn ärgerlich und sagte: "Lals ihna. Ich trat vor ihn. Er antwortete und sagte zu mir: "Sieh, den Auftrag, den meine Văter vordem ausgeführt haben, habe ich auch ausgeführt, obschon du mir nicht das gethan hast, was deine Văter mir gethan haben ${ }^{5}$ ). Sieh, (auch) das Letzte deines Holzwerks ist angekommen und liegt da. Thue nun nach meinem Wunsch und komme, es einzuladen, denn wahrlich, man giebt es dir.

50 Komme") (aber) nicht, die Schrecklichkeit des Meeres zu betrachten; (oder) wenn du dir (doch) die Schrecklichkeit des Meeres betrachtest, so betrachte auch meine eigene ${ }^{7}$ ). Wahrlich, ich habe dir nicht das gethan, was man den Gesandten des Cha-em-wese gethan hat, als sie 15 Jahre in diesem Lande blieben ${ }^{8}$ ). Sie starben, wo sie waren^. Er sagte zu seinem Truchsefs: - Führe ihn und lafs ihn ihr Grab, in dem sie ruhen, betrachten *.

1) Lies: sie sandten(?), er erhält also auch noch ein persönliches Geschenk.

2) Was hic soll, weifs ich nicht.

) Oder Schirmes? Ein Fremdwort srpt, den ungefähren Sinn ergiebt das Determinativ T Ägypter.

4) Über diesen uns unverständlichen Witz vergl. oben S. 2. Pen-Amon ist natürlich ein

5) Also auch jetzt noch betrachtet der Fürst sich als schlecht oder gar nicht bezahlt.

- So wörtlich, es wird eine Redensart sein, wie unser - Komme mir nicht damit, dafs du ... *

7) Twr qwwt. Der Sinn ist: Nun mache aber auch, dals du fortkommst und bleibe nicht etwa aus Angst vor der See noch hier, sonst geht es dir schlimm.

s) Was ist diesen Gesandten des $H c-m-203 s t$ geschehen? Hat man sie nicht nach Hause gelassen? Oder blieben sie auf eigene Hand in Byblos und gingen dort zu Grunde? - Bei Hc-mwosst handelt es sich wohl nicht um den bekannten Prinzen, denn dessen Boten würden Gesandte Ramses' II. heifsen. Vielleicht darf man daran denken, dafs Ramses IX. und Ramses XII. diesen Beinamen in ihrem zweiten Schild führen; dann könnte es sich um eine Gesandtschaft des ersteren handeln. 
Ich sagte: "Lafs es mich nicht betrachten. Bei Cha-em-wese waren es Menschen, die er dir als Gesandte schickte; aber Menschen ....... und es war kein [Gott] unter seinen Boten. (Und doch) sagst du: Geh und sieh dir deine Genossen an ${ }^{1}$ ).

Freust du dich nicht (vielmehr) $\left.{ }^{2}\right) \mathbf{n} 56$ und lä (st du dir nicht einen Denkstein machen und sagst auf ihm: "Amon-Re, der Götterkönig, hat ${ }^{3}$ ) mir den Amon des Weges, seinen [gőttlichen] Boten gesandt nebst dem Wen-Amon, seinem menschlichen Boten, wegen des Holzwerks des grofsen herrlichen Schiffes des Amon-Re, des Götterkőnigs. Ich habe es gefällt, ich habe es eingeladen, ich habe ihn mit meinen Schiffen und meiner Mannschaft ausgerustet ${ }^{*}$ ), ich habe sie nach Ägypten kommen lassen, um für mich bei Amon 10000 Jahre des Lebens zu erflehen noch hinzu zu dem mir bestimmten (Leben), und so ist es geschehen. ${ }^{5}$ ). Wenn dann zu anderer Zeit ein Bote aus dem Lande Ägypten kommt, der die Schrift kennt, und er liest deinen Namen auf dem Denkstein, so wirst du Wasser im Westen empfangen gleich den Göttern, die ${ }^{60}$ hier $^{\circ}$ ) sind. .

Er sagte zu mir: "Das ist ein grofses Zeugnis ${ }^{7}$ ), was du zu mir gesagt hast..

Ich sagte zu ihm: - Was nun das Viele anbetrifft, was du mir gesagt hast ${ }^{8}$ ), wenn ich zu dem Wohnort des Hohenpriesters des Amon komme und er deinen Auftrag in deinem Auftrag sehen wird ${ }^{\natural}$ ), so wird $[\mathrm{er}]^{10}$ ) dir etwas herbeischaffen lassen .

Ich ging am Strande des Meeres bis dahin, wo die Balken lagen, und ich erblickte elf Schiffe, die auf dem Meere kamen und den Zakar gchörten (und mit dem Auftrag kamen)"1): Verhaftet ihn, lafst kein Schiff von il/m ${ }^{12}$ ) nach dem Lande Ägypten.

Da setzte ich mich hin und weinte. Der Briefschreiber des Fürsten kam zu mir heraus. ${ }^{6} \mathrm{Er}$ sagte zu mir: "Was hast du?. Ich sagte zu ihm: $D$

1) Sinn: Unsere Gesandtschaft besteht aus einem Gott, da sind das nicht unsere Genossen.

2) Anstatt so ärgerlich auf mich zu sein.

2) Bei dieser Inschrift, die Wen-Amon entwirft, wendet er die ălteren Formen ohne Hülfsverb im selbständigen Satz an, um feierlich zu sprechen.

4) Danach mufs man annehmen, dafs der Fürst dem Wen-Amon auch so zu Hülfe kommen will. Auch aus II 82 geht hervor, dals er nacliher wirklich mit einem Schiffe des Finrsten von Byblos heimfährt.

1) Steht wirklich mto hprf, wie Golmmscherf giebt, so hat Wen-Ainon diese Unform gebildet, weil ihm mhof bpr. riguguwre zu vulgär schien.

2) Man erwartet -dort. (im Totenreich), doch weifs ich nicht, ob man $\underset{8}{\longrightarrow}$ so gebrauchen kann. Bemerkenswert ist, daf́s bei deın Fürsten Verständnis fur den ägyptischen Totenkult und seine Ausdrücke und Formen vorausgesetzt wird.

7) Ist das Ernst oder Ironie?

8) D. h.: Deine Klagen wegen des Geldes.

9) Der Satz ist wohl entstellt (lies etwa: Deine Krfüllung seines Auftrags(?)).

10) Die Ergänzung •er: verdanke ich W. Max MǘLra.

11) In Text ist dieses alles nur mit einem xe ausgedrūckt; ăhnlich, wenn auch nicht ganz so arg, 1136.69.

19) Wie orugnpe firsag; ähnlich II 83. 
siehst doch die Vögel'), die wieder(?) nach Ägypten ziehen. Sieh sie an, sie gehen zum kühlen Teich, und bis wann(? $\left.)^{-}\right)$bleihe ich hier verlassen? Denn du siehst doch die, welche kommen, mich wieder ${ }^{3}$ ) zu verhaften .

Er ging weg und sagte es dem Fürsten. Der Fürst fing zu weinen an wegen der Worte, die man ihm sagte und die so traurig waren. Er schickte. seinen Briefschreiber zu mir heraus, und der l,rachte mir zwei mśh Wein und einen Widder $\left.(?)^{4}\right)$. Er schickte mir die Tent-Nawt, eine ägyptische Săngerin, die bei ihm war, und sagte ihr: "Singe ihm; er fange keine Grillen "). Er liels mir sagen: II 70,Ifs und trink und fange keine Grillen. Morgen wirst du alles hören, was ich sagen werde.

Als es Morgen war, liels er (die Leute) in seinen ...... rufen und trat in ihre Mitte und sagte zu den Zakar: -Was soll es, dafs ihr kommt? Sie antworteten ihm: - Wir werden die ganz zerschlagenen ${ }^{\circ}$ ) Schiffe verfolgen, die du nach Ägypten mit unseren ....... -genossen ${ }^{7}$ ) schickst... Er sagte zu ihnen: - Ich kann den Gesandten des Amon nicht in meinem Lande verhaften. Lafst ihn mich absenden und verfolgt ihn (dann), um ihn zu verhaften .

Er schiffte mich ein und sandte mich ab .... dem Hafen des Meeres. Der Wind trieb mich nach dem Lande ${ }^{75}$ Alaschija $^{8}$ ). Die von der Stadt zogen heraus gegen mich, um mich zu töten. Ich wurde zwischen ihnen zum Wohnsitz der $\not H 3-t i-b 3$, der Fürstin der Stadt, geschleppt(?). Ich traf sie, wie sie aus ihrem einen Hause ${ }^{9}$ ) herauskam und in ihr anderes eintrat. Ich begrülste sie und sagte zu den Leuten, die bei ihr standen: Es giebt gewils einen unter euch, der Ägyptisch versteht. Einer von ihnen sagte: "Ich verstehe es . ${ }^{10}$ ). Ich sagte zu ihm: -Sage meiner Herrin: Bis nach 'Theben, nach dem Wohnsitz des Amon hin, hatte ich gehört: in allen Städten thut man Unrecht,

1) Es ist eine bestimmte Sorte, g3ł, gemeint. Das - wieder: ist fraglich, wörtlich heilst es: -zweimal *.

2) Was hier steht, entspräche einem ganteagg er -bis welche Sache kommt?- Aber kann man einen solchen Satz vor den Hauptsatz stellen?

s) Danach haben ihn die Zakarleute schon einmal verhaftet gehabt.

4) '/3-yro-r's steht, wie GolenischefF gesehen hat, wohl für br.

s) -Sein Herz nehme nicht Gedanken.:

6) Oder geprügelten-, wohl nur eine despektierliche Bezeichnung der Schiffe.

$\left.{ }^{7}\right)$ Das Wort titi ist nach Abb. 5, 22 ein Wort des Redens mit irgend einem Beigeschmack. Sind die -titi-genossen. daher nicht vielleicht einfach die Mitbarbaren? Die Zakarleute geben vor, es sei schon Unrecht, dafs der Fürst seine kananäischen Matrosen zu deın verhalsten Ägypten schicke.

s) Ägyptisch $i 3-r 3-s\}$, d. h. ' $r s$, ich benutze die aus den Tellamarnatexten bekannte keilschriftliche Form, die wenigstens aussprechbar ist. Über das Land steht fest und auch unser Text lestätigt es, dafs es bei dem nördlichen Syrien liegt; die von Il. Winckler gegebene Deutung auf Cypern scheint auch mir sehr wahrscheinlich.

${ }^{9}$ ) Wohl nicht das alte Wort roc -der Palast., sondern wie auch Goceniscrefr es gefalst hat: $p 3$ we $p r$.das eine Haus*.

iv) In Alaschija spricht man also eine andere Sprache als in Phönizien, und das Ägyptische liegt den Alaschijaleuten ganz fern. 
im Lande Alaschija thut man Recht ${ }^{1}$ ). Und nun thut man auch hier täglich Unrecht! Sie sagte: -Was soll das, $1080 \mathrm{dafs}$ du so redest? Ich sagte zu ihr: -Wenn das Meer wütend war und der Wind mich zu dem Lande verschlagen hat, in dem du lebst, so wirst du nicht erlauben, dafs sie mein ......2) fassen, um mich zu töten, da ich doch ein Bote des Amon bin. Sieh wohl zu, nach mir wird man $^{3}$ ) immerfort suchen. Diese Mannschaft des Fürsten von Byblos ${ }^{1}$ ), die sie töten wollen ${ }^{5}$ - - wahrlich, wenn ihr Herr zehn Mannschaften von dir antrefien wird, so tötet er sie auch..

Sie liefs die Leute herbeirufen, und man stellte sie hin. Sie sagte zu mir: - Leg dich schlafen ....

Damit bricht der Papyrus ab, und falls die verlorene Seite nicht unerwartet noch irgendwo auftaucht, wird unsere Neugierde über Wen-Amons weitere Schicksale nie befriedigt werden. Nehmen wil an, dafs es ilım gelungen ist, heil mit seinem Schiffe aus Alaschija zu entkommen und dafs er auch den lauernden Zakar entgangen ist, so wird sein Empfang in Theben doch kein allzu freudiger gewesen sein. Denn er hrachte zwar das gewünsclite Holz mit, aber umsonst hatte er es doch nicht hekommen, und es lag Hrihor noch ob, dem habgierigen Phönizier auch "das Jämmerliche^, die zugesagte Bezahlung, zu schicken.

1) Klingt wie ein Citat aus einem Lied oder wie ein Sprichwort.

2) Wörtlich: - mein Vorderteila; man könnte es von dem Fıntern des Schiffes verwtehen. doch ist er ja aın Land angegrlffen worden. I's liegt also wohl nur eine Redensart fïr überfallen oder ähnliches vor.

3) D. h.: ich bin ein vornehıner Jann, den ihr nicht unbernerkt und straflos aus der Welt schaffen könnt. -110 die wohl nur graphisch daraus entartet ist.

4) I). L.: die Schiffsmannschaft, die Wen-Amon und das Holz fährt.

5) El meint ccoregy-gorhc; dafs das sic hier, wie nicht selten, ungenaue Schreibung des Suffexes $s$ ist, ergiebt sich schon daraus, dafs ein sic als Pronomen absolutum ja nicht vom Infinitiv abhüngen dïrfte. Wen-Aınon droht also hier auch mit dem Zorne des Fïrsten von Byblos; den mochte man in Alaschija wohl inehr fürchten als den Zorn des fernen Ägypten. 